\title{
Intersection of Reward and Memory in Monkey Rhinal Cortex
}

\author{
Andrew M. Clark, Sebastien Bouret, Adrienne M. Young, and Barry J. Richmond \\ Laboratory of Neuropsychology, National Institute of Mental Health, National Institutes of Health, Department of Health and Human Services, Bethesda, \\ Maryland 20892
}

In humans and other animals, the vigor with which a reward is pursued depends on its desirability, that is, on the reward's predicted value. Predicted value is generally context-dependent, varying according to the value of rewards obtained in the recent and distant past. Signals related to reward prediction and valuation are believed to be encoded in a circuit centered around midbrain dopamine neurons and their targets in the prefrontal cortex and basal ganglia. Notably absent from this hypothesized reward pathway are dopaminergic targets in the medial temporal lobe. Here we show that a key part of the medial temporal lobe memory system previously reported to be important for sensory mnemonic and perceptual processing, the rhinal cortex $(\mathrm{Rh})$, is required for using memories of previous reward values to predict the value of forthcoming rewards. We tested monkeys with bilateral Rh lesions on a task in which reward size varied across blocks of uncued trials. In this experiment, the only cues for predicting current reward value are the sizes of rewards delivered in previous blocks. Unexpectedly, monkeys with Rh ablations, but not intact controls, were insensitive to differences in predicted reward, responding as if they expected all rewards to be of equal magnitude. Thus, it appears that Rh is critical for using memory of previous rewards to predict the value of forthcoming rewards. These results are in agreement with accumulating evidence that Rh is critical for establishing the relationships between temporally interleaved events, which is a key element of episodic memory.

\section{Introduction}

The predicted value of a reward depends upon numerous factors, including its magnitude and the likelihood it will be obtained (Tversky and Kahneman, 1981). Predicted value is also dependent upon the context in which a reward is presented (Tversky and Kahneman, 1981). For example, the value of winning $\$ 100$ on a spin of the roulette wheel varies according to whether you won $\$ 10$ or $\$ 1000$ on the previous spin. This contextual modulation of predicted value has been shown to affect behavior in humans and several other species (Lowe et al., 1974; Perone and Courtney, 1992; Williams et al., 2011).

There are several reasons the rhinal cortex $(\mathrm{Rh})$, an important part of the medial temporal lobe (MTL) memory system in rats, monkeys, and humans (Eichenbaum et al., 2007), is an attractive candidate for the neural substrate of this contextual modulation

Received Feb. 23, 2012; revised March 19, 2012; accepted March 27, 2012.

Author contributions: A.M.C., S.B., and B.J.R. designed research; A.M.C. and A.M.Y. performed research; A.M.C. and B.J.R. analyzed data; A.M.C., S.B., and B.J.R. wrote the paper.

This work was supported by the Intramural Research Program of the National Institute of Mental Health. We thank Dr. R. Saunders for making and assisting in reconstructing the rhinal lesions as well as for comments during development of this manuscript, Drs. M. Eldridge, L. Optican, and M. Mishkin for their comments during development of this manuscript, M. Malloy for assistance in lesion reconstructions, and R. Reoli for assistance in obtaining MR images. The opinions expressed in this article are the authors' own and do not reflect the view of the U.S. National Institutes of Health, the Department of Health and Human Services, or the United States government.

Correspondence should be addressed to Dr. Barry J. Richmond, National Institutes of Health, 49 South Convent Drive, Bethesda, MD 20892. E-mail: bj@@In.nimh.nih.gov.

S. Bouret's present address: Team Motivation Brain and Behavior, ICM-Institute du Cerveau et de la Moelle épinière, Hôpital Pitié-Salpêtrière, 47, boulevard de I'Hôpital, 75013 Paris, France.

A. M. Young's present address: Department of Visual Neuroscience, UCL Institute of Ophthalmology, University College London, 11-43 Bath Street, London EC1V 9EL, UK.

DOI:10.1523/JNEUROSCI.0887-12.2012

Copyright $\odot 2012$ the authors $\quad 0270-6474 / 12 / 326869-09 \$ 15.00 / 0$ of predicted value. First, deficits in visual stimulus-stimulus associations (Murray et al., 1993), cross-modal associations (Parker and Gaffan, 1998; Goulet and Murray, 2001), and object recognition memory (Meunier et al., 1993; Mumby and Pinel, 1994; Yonelinas et al., 1998) following Rh ablations suggest Rh is critical for many types of memory. Second, deficits in using sensory (Buckmaster et al., 2004; Sauvage et al., 2010) or environmental context (Bucci et al., 2000; Burwell et al., 2004; Moser et al., 2008) to guide behavior have been observed following Rh lesions. Third, $\mathrm{Rh}$ is rich in dopamine (Akil and Lewis, 1993), a neuromodulator strongly linked to reward processing (Schultz, 2007). Finally, the integrity of the rhinal cortex (Murray et al., 1998; Liu et al., 2000; Winters et al., 2010)—and its local dopamine neurotransmission (Liu et al., 2004) — are necessary for using visual cue-reward associations to adjust motivation.

Despite this evidence for a widespread role of $\mathrm{Rh}$ in both memory and reward guided-behavior, current theories of Rh function posit that this structure plays an exclusive role either in recognizing whether current stimuli have been previously encountered (Brown and Aggleton, 2001) and/or in high-level (primarily visual) perception (Murray et al., 2007). In both frameworks, alterations of reward-guided behavior following $\mathrm{Rh}$ ablations spring from an impairment in forming or maintaining cue-reward associations. We wondered whether Rh might play a broader role in determining predicted reward value. Specifically, we hypothesized that $\mathrm{Rh}$ is important when estimating predicted reward value requires comparisons between forthcoming and remembered rewards.

Accordingly, we tested monkeys with bilateral Rh ablations on two tasks. In the first (visually cued task; Fig. $1 B$ ), in a replication 
A

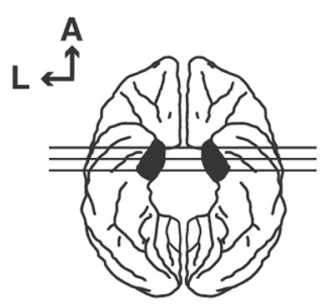

Ventral View
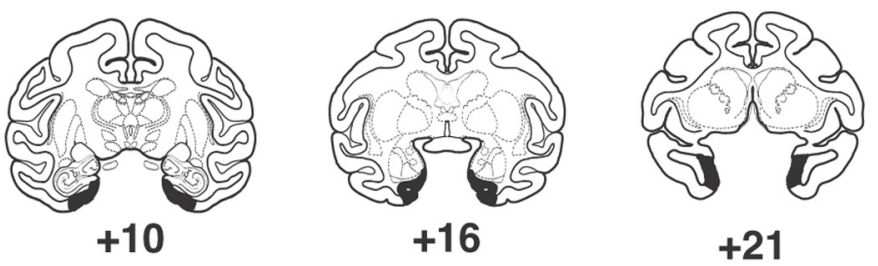

\section{B}

\section{Visually cued task}
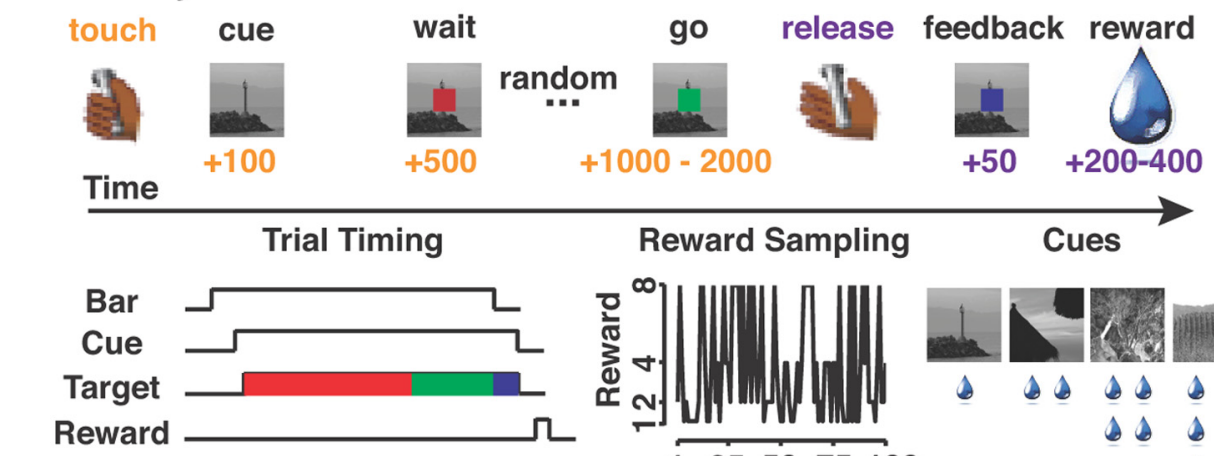

C
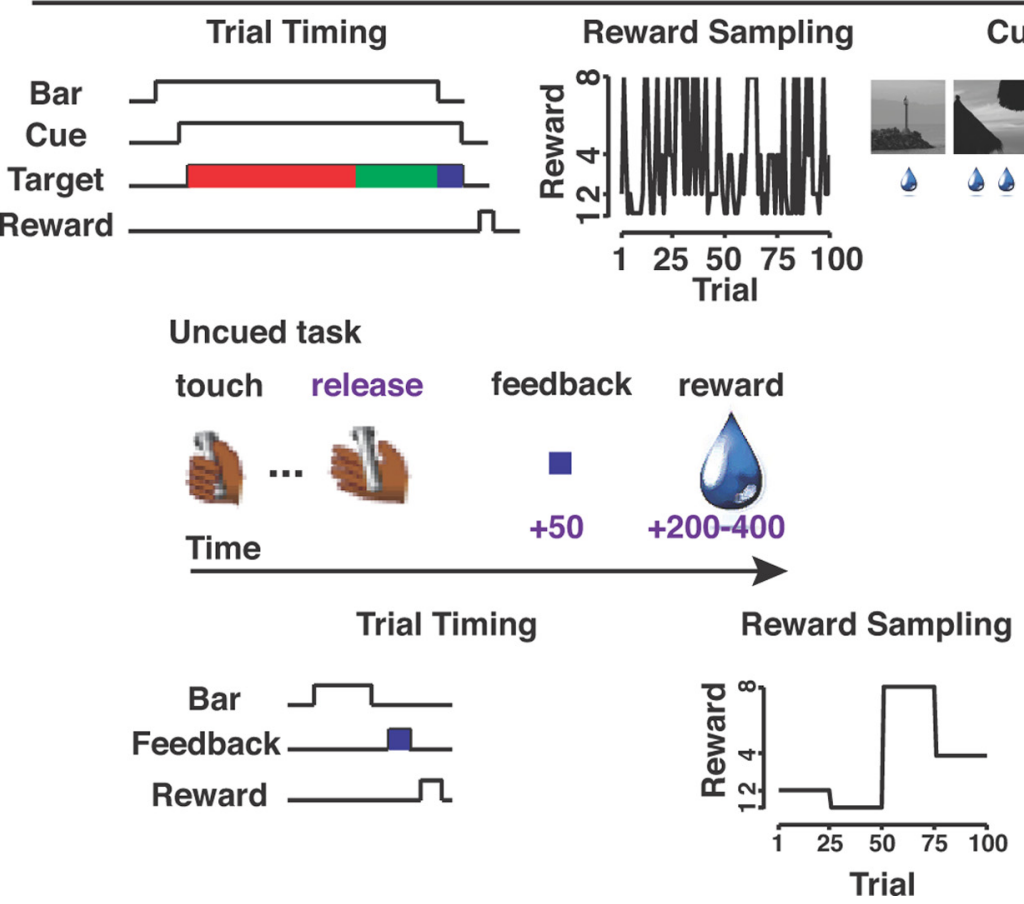

feedback reward
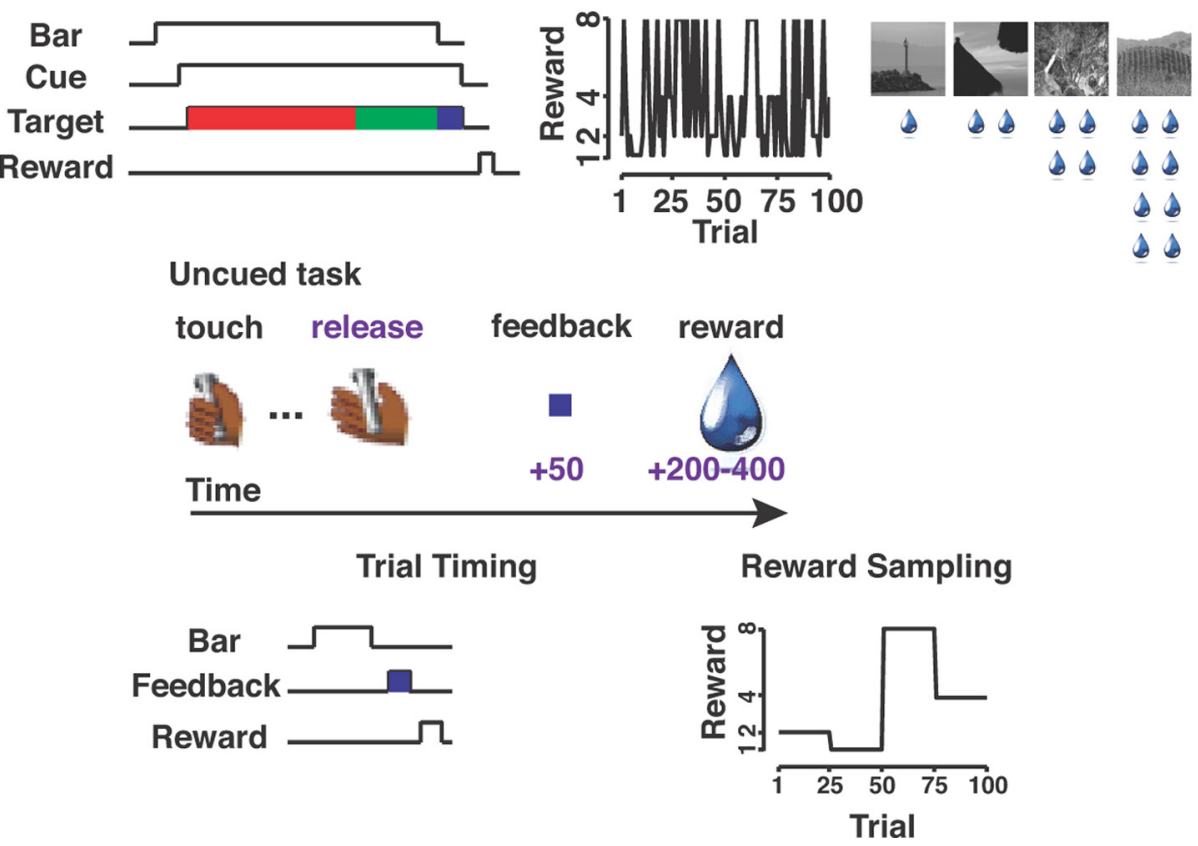

Figure 1. Experimental logic and design. $\boldsymbol{A}$, Intended lesions. $\boldsymbol{B}, \boldsymbol{C}$, Schematic illustration of the behavioral tasks. In the visually cued task, a specific visual cue presented on each trial indicated the amount of fluid reward (1,2,4, or 8 drops) to be delivered upon correct completion of a red-green color discrimination. Reward size was varied randomly trial-by-trial (error trials repeated until completed correctly). In the uncued task, rewards were delivered for touching and releasing a lever, monkeys were free to initiate responses at their own pace. Reward size was varied block-wise in this task (1, 2, 4, or 8 drops, 25 responses per block). For each task, event timing is referenced relative to bar touch or release (color code).

of previous studies, visual cues predicted reward size. In the second (uncued task; Fig. $1 C$ ) the relationship between reward sizes obtained in the recent and distant past was the only source of information for predicting and valuing rewards. In both tasks, the sensory and motor demands were constant across trials, thus, variations in performance were interpreted as an indication of the monkeys' valuation of a predicted reward.

\section{Materials and Methods}

Subjects

We tested six male rhesus monkeys (Macaca mulatta), weighing between 5 and $14 \mathrm{~kg}$, as subjects in this study. Three monkeys served as unoperated controls and three monkeys were given bilateral Rh lesions. All lesions were made before all behavioral training and testing. All experimental procedures conformed to the National Institutes of Health Guide for the Care and Use of Laboratory Animals and were approved by the National Institute of Mental Health Animal Care and Use Committee.

\section{Surgery}

Aspiration lesions of Rh were performed as described previously (Fritz et al., 2005), we restrict our description here to a few key points. Bilateral lesions were performed within a single surgery. Surgeries were performed under aseptic conditions in a fully equipped operating suite with veterinary supervision. Before surgery, animals were sedated with ketamine hydrochloride $(10 \mathrm{mg} / \mathrm{kg}$, i.m.); a surgical level of anesthesia was then induced and maintained with isoflurane gas ( $2-4 \%$ to effect). Body temperature, heart rate, blood pressure and expired $\mathrm{CO}_{2}$ were monitored throughout all surgical procedures. After removal of a bone flap and reflection of the dura mater, intended lesion boundaries were marked via electrocautery and tissue was then removed through a combination of 


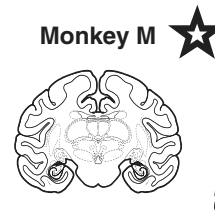

$+8$

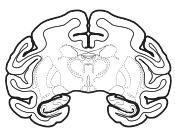

$+11$

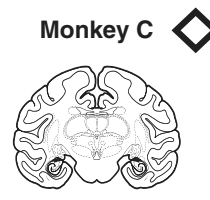

$+8$

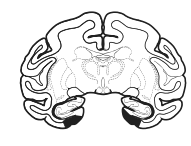

$+11$

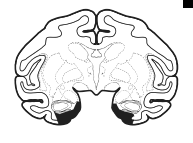

$+14$

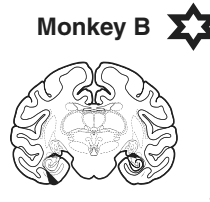

$+8$
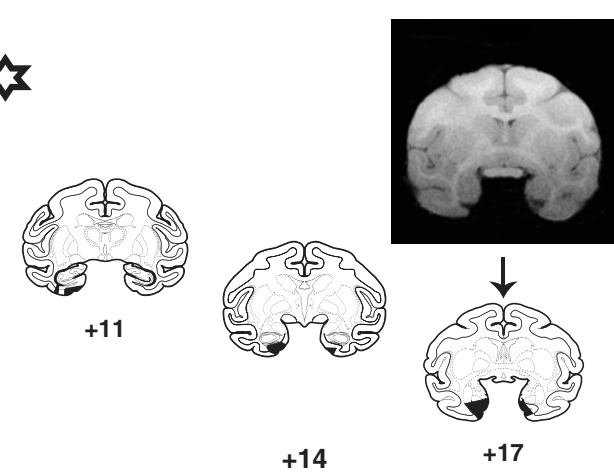

$+14$

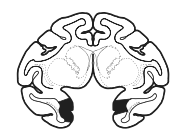

$+20$

Figure 2. Bilateral aspiration lesions of rhinal cortex. Estimates of the extent of aspiration lesions for the three monkeys in the Rh group are plotted on coronal sections at the levels indicated. Lesions were reconstructed using MR images (MR scans were done at $3 \mathrm{~T}$ for monkeys $B$ and $M$ and $1.5 T$ for monkey C). Arrows connect a representative MR image for each monkey to the corresponding section. The symbols used to plot data from individual monkeys in the panels of Figures 3 and 4 are shown next to the reconstruction from each monkey. For the most part, lesions were restricted to $\mathrm{Rh}$, with damage to adjacent structures distributed idiosyncratically across monkeys, and, within each monkey, across hemispheres.

suction and electrocautery using a fine gauge metal pipette with the aid of an operating microscope (Zeiss).

\section{Lesion reconstruction}

Intended lesions are shown on a ventral view of the macaque brain and on coronal sections at the indicated levels in Figure $1 \mathrm{~A}$. The intended lesion subsumed both the entorhinal (Brodmann's area 28) and perirhinal subdivisions (Brodmann's areas 35 and 36), encompassing the cortex within $2 \mathrm{~mm}$ medial and lateral to the rhinal sulcus.

The full extent and location of all lesions was assessed using T1 weighted magnetic resonance (MR) image scans ( $1 \mathrm{~mm}$ slices, $0.4 \mathrm{~mm}$ in-plane resolution, either 1.5 or $3 \mathrm{~T}$ ). It has been shown that estimates of lesion boundaries and volumes based upon this method are consistent with estimates based upon standard histological techniques (Bachevalier et al., 1999; Liu et al., 2000). To reconstruct lesions, coronal MR images were first matched to coronal plates in a stereotaxic rhesus monkey brain atlas; following alignment, lesion boundaries were marked on each plate. After determining the boundaries of the lesion in each section, the full extent of the lesion was interpolated across sections. Representative reconstructions for all monkeys in the $\mathrm{Rh}$ group are shown in Figure 2 .

\section{Tasks and training}

During all testing sessions, monkeys squatted in a primate chair inside a darkened, sound-attenuated testing chamber. They were positioned $57 \mathrm{~cm}$ from a computer monitor subtending $40 \times 30$ degrees of visual angle. Task timing and visual stimulus presentation were under the control of networked computers running, respectively, custom written (Real-time Experimentation and control, REX) and commercially available software (Presentation, Neurobehavioral Systems) for the design and control of behavioral experiments.

Red-green color discrimination. Monkeys were initially trained to grasp and release a touch-sensitive bar to earn fluid rewards. After this initial shaping, we introduced a red-green color discrimination task (Bowman et al., 1996). Red-green trials began with a bar press, $100 \mathrm{~ms}$ later a small red target square $\left(0.5^{\circ}\right)$ appeared at the center of the display, superimposed on a white noise background. Animals were required to continue holding the touch bar until-at a random time between 500 and $1500 \mathrm{~ms}$ later-the color of the target square changed from red to green. Rewards were delivered if the animal released the bar between 200 and $1000 \mathrm{~ms}$ after the color change, releases occurring either before or after this epoch were counted as errors. All correct responses were followed by visual feedback (blue square) $50 \mathrm{~ms}$ after bar release and reward delivery $200-400 \mathrm{~ms}$ after visual feedback.

Visually cued task. After an animal reached criterion in the red-green task (3 consecutive sessions with $>85 \%$ correct performance) we introduced a visually cued reward size task (Fig. 1 B; Minamimoto et al., 2009). Each trial began when animals grasped the touch bar. Instead of the appearance of the red target stimulus, bar press was now initially followed (after $100 \mathrm{~ms}$ ) by the presentation of a cue image (grayscale natural images, $10^{\circ} \times 10^{\circ}$; Fig. $1 \mathrm{~B}$ ). Each cue signaled which of four different reward sizes (1,2, 4 or 8 drops, random draw) the animal would earn upon successful completion of the trial. $400 \mathrm{~ms}$ after cue presentation the red target square appeared, now centered on the cue. Animals were once more required to hold the touch bar until the target square color changed from red to green (500$1500 \mathrm{~ms}$ ). Releases that occurred outside of the 200-1000 ms interval following the color change were counted as errors; error trials were repeated until completed correctly. Successful bar releases were signaled via visual feedback (blue square). Reward delivery followed feedback by 200-400 ms and lasted for between 150 and $2500 \mathrm{~ms}$ (200 ms interdrop interval). Periodically, the reward system was calibrated to ensure an average drop size of $0.1 \mathrm{ml}$.

Monkeys were tested for 15 sessions using the same set of four cue images. Monkeys were tested in the visually cued task for 1 session per day ( $2 \mathrm{~h}$ per session), 5-6 d per week.

Uncued task. In contrast to the red-green color discrimination and visually cued reward size task, the uncued task (Fig. 1C) contained neither visual cues to reward size nor response cues that the monkey was required to attend to, to successfully complete a trial. To earn a reward, 
monkeys simply had to touch and release a bar. Every bar release that did not occur during the feedback or reward periods was followed by visual feedback (a blue spot, $50 \mathrm{~ms}$ after release) and a reward $(1,2,4$, or 8 drops, 200-400 ms after visual feedback). To provide the monkeys with a basis for predicting upcoming reward size, reward sizes were varied in blocks (random draw), with 25 trials per block. To distinguish the uncued from the visually cued task we displayed a different background image on the computer monitor during the testing session. The duration of an uncued session was adjusted for each monkey to approximately equate the total volume of reward earned across the visually cued and uncued tasks. Because of the additional trial time for presentation of the cue image and red-green target images in the visually cued task, rewardto-reward intervals were considerably longer in the visually cued versus the uncued task, and thus uncued sessions were shorter. Following 15 sessions of testing on the visually cued task, monkeys were run in the uncued task for 15 sessions ( 1 session per day, 5-6 d per week).

\section{Data analysis}

All data analysis was performed using custom software written in Matlab (Mathworks) and R (Team, 2004).

The visually cued task contained a target signal (red-green color change) that monkeys had to respond to (with a bar release) to earn fluid reward. Thus, for this task, we were able to calculate both error rates and reaction times. To assess the effect of reward size on performance, error rates and reaction times were calculated for each session: (1) separately according to reward size using all trials in a session, and (2) after binning trials according to reward size and normalized accumulated reward (defined as the accumulated amount of reward earned at a particular time in the session divided by the total volume of reward earned in the session).

We used the interval between the points when monkeys touched and released the bar (release interval, RI) as our measure of performance in the uncued task. To facilitate comparisons between the visually cued and uncued tasks, we excluded release intervals that exceeded the maximum duration of a visually cued trial. Taking the logarithm of the release interval, excluding release intervals that were greater than the SD by a factor of 5, or examining the median release interval yielded similar results. Only the results obtained using the first metric are presented here.

To analyze the dynamics of behavior in the uncued task, we first normalized release intervals to the mean and SD in each session, we then averaged across within-block trial numbers within each session, across sessions for each monkey and across monkeys for each experimental group. Finally, we reordered these standardized release intervals so that responses faster than the mean assumed positive values and responses slower than the mean assumed negative values.

ANOVA models were used to evaluate whether the factors: reward size, normalized accumulated reward, session number, surgical treatment, or within-block trial number had a significant effect on performance. Because baseline error rates and release intervals vary across subjects, the variability accounted for by individual monkeys was included as an error term within each ANOVA model. Mixed-model ANOVAs were used for comparing experimental groups.

\section{Results}

We tested a group of monkeys with bilateral aspiration lesions of Rh $(n=3$, Figs. $1 A, 2)$ as well as a group of unoperated controls $(n=3)$ on two simple instrumental tasks. In the first task, in a replication of prior studies (Liu et al., 2000, 2004), reward size was explicitly signaled on each trial by a specific visual stimulus (Fig. $1 B$ ). In the second task, reward size simply varied across blocks of trials (Fig. 1C).

\section{Visually cued reward size task}

In the visually cued reward size task monkeys were rewarded for releasing a bar when the color of a visual target changed from red to green (see Material and Methods, Visually cued task). On each trial a specific visual cue signaled the amount of reward available upon correct completion of the trial. Errors could fall into one of
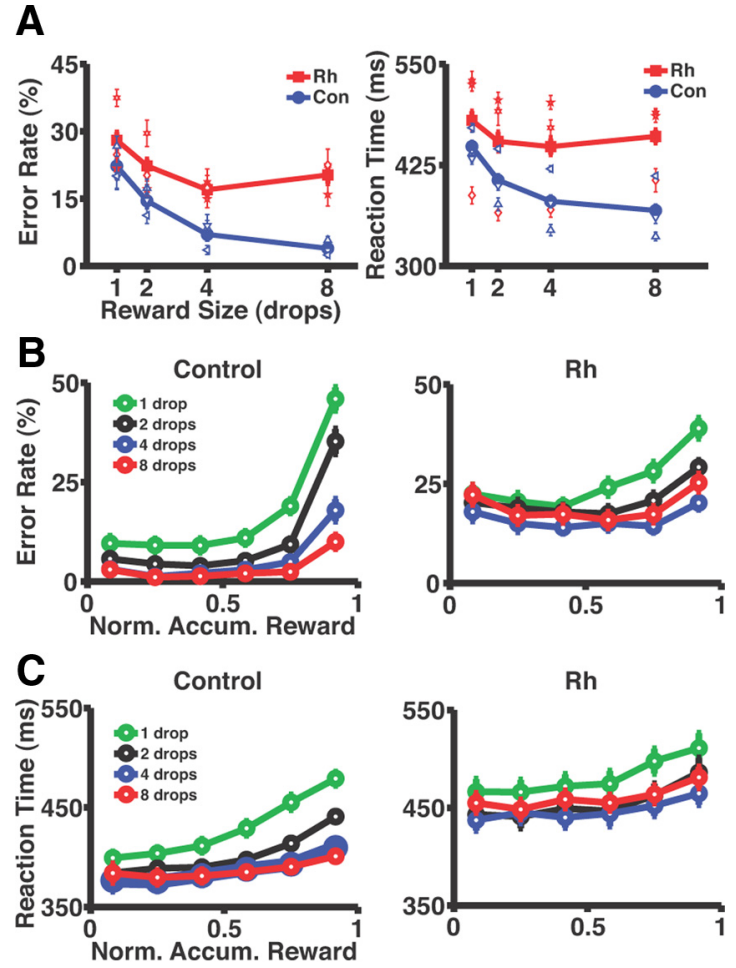

Figure 3. Rh ablations disrupt cue-reward associations. $\boldsymbol{A}$, Control (Con) and Rh group performance of the visually cued task is plotted as error rates (left) or reaction times (right, ordinate) versus reward size (abscissa). Filled symbols, Group means; open symbols, individual monkeys. There was a significant reward size by lesion interaction. $\boldsymbol{B}$, Average error rates (ordinate) are plotted versus normalized accumulated reward (abscissa; see Materials and Methods, Data analysis, for calculation) separately for trials ending in different reward sizes. Left, Control group; right, Rh group. $\boldsymbol{C}$, Conventions as in $\boldsymbol{B}$ but for reaction time data. There was a significant three-way interaction between group, reward size, and normalized accumulated reward. In all panels, error bars indicate SEM. Norm, Normalized; Accum, accumulated; ms, milliseconds.

two categories: early (before the red-green change) or late ( $>1000 \mathrm{~ms}$ after the red-green change) bar releases. There was no statistically significant difference in the proportions of error types for the control (error type: $F_{(1,4)}=0.11, p=0.77$ ) or Rh groups (error type: $F_{(1,4)}=3.8, p=0.12$ ).

As seen previously (Minamimoto et al., 2009), the control group's performance in the visually cued task was significantly affected by reward size, with error rates and reaction times both decreasing with increasing reward size and increasing with accumulated reward (Fig. $3 A-C$; error rates: reward size, $F_{(3,6)}=66.6$, $p<10^{-5}$, reward size $\times$ accumulated reward, $F_{(15,30)}=13.4, p<$ $10^{-8}$; reaction times: reward size, $F_{(3,6)}=6.6, p=0.03$, reward size $\times$ accumulated reward, $\left.F_{(15,30)}=9.8, p<10^{-7}\right)$. Additionally, as reported before (Liu et al., 2000), bilateral aspiration lesions of Rh significantly altered performance when visual cues signaled reward value (Fig. $3 A-C$ ). That is, following $\mathrm{Rh}$ ablations, monkeys were less sensitive (as measured by both error rates and reaction times) to the reward size signaled by the visual cues (error rates: group, $F_{(1,4)}=9.9, p=0.03$, reward size $\times$ group, $F_{(3,12)}=5.5, p=0.01$, reward size $\times$ accumulated reward $\times$ group, $F_{(15,75)}=9.3, p<10^{-11}$; reaction times: group, $F_{(1,4)}=1.9, p=0.23$, reward size $\times$ group, $F_{(3,12)}=5.2, p=0.02$, reward size $\times$ accumulated reward $\times$ group, $F_{(15,75)}=5.6, p<$ $\left.10^{-6}\right)$. Although impaired, the Rh group did still exhibit a weak residual sensitivity to reward size (error rates: reward size, $F_{(3,6)}=$ $5.4, p<0.04)$. 
A
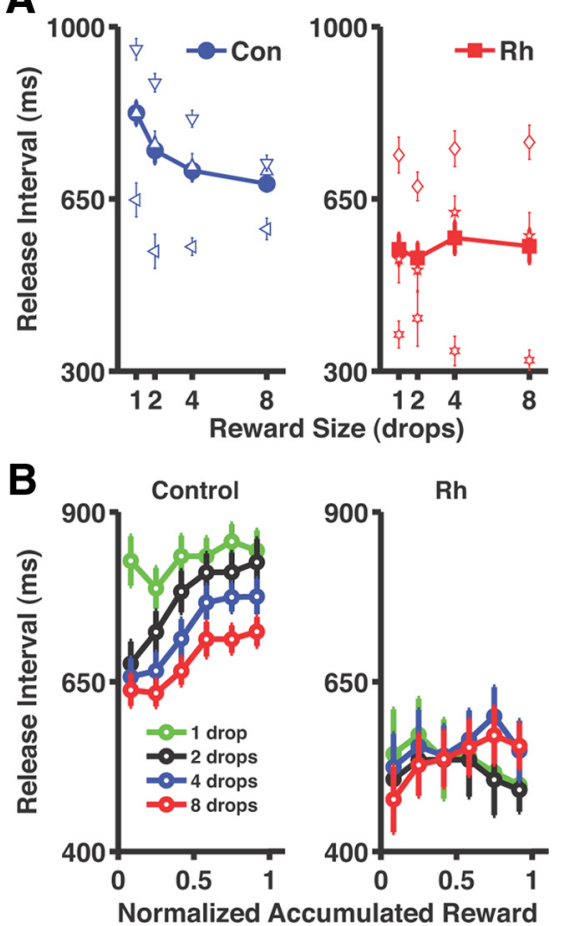

Figure 4. Rh ablations abolish the ability to use comparisons between current and previous rewards to estimate predicted value. $A$, Control and Rh group performance in the uncued task is plotted as the mean interval between touching and releasing the lever (release interval, ordinate) versus reward size (abscissa). Filled symbols, Group means; open symbols, individual monkeys. Left, Control group; right, Rh group. There was a significant reward size by lesion interaction. $\boldsymbol{B}$, Average release intervals (ordinate) are plotted versus normalized accumulated reward (abscissa) separately according to reward size. Left, Control group; right, Rh group. There was a significant main effect of both reward size and accumulated reward for the control group.
Control

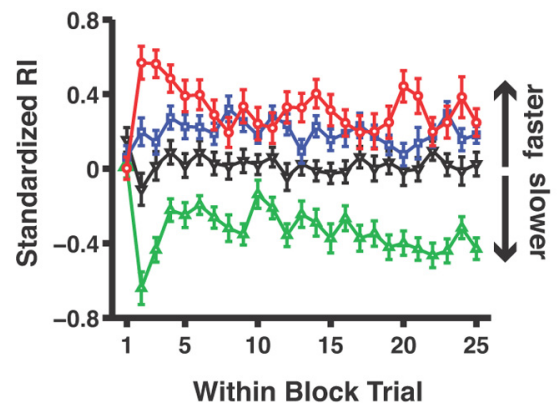

\section{$\mathbf{R h}$}

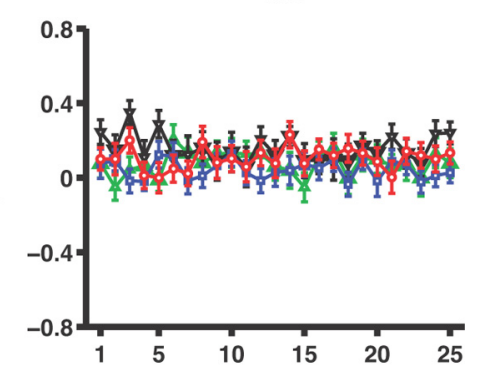

(and thus, in the total volume of reward earned in a session; lesion $\times$ bar presses, $\left.F_{(1,4)}=0.01, p=0.9\right)$. This suggests the groups were equally motivated to perform the task and understood that bar releases led to reinforcement.

The control group's responses in the uncued task were significantly affected by reward size and accumulated reward. Specifically, the interval between bar touch and release (release interval) decreased with increasing reward size and increased with accumulated reward (Fig. $4 A, B$; reward size, $F_{(3,6)}=33.1, p<10^{-4}$; accumulated reward, $\left.F_{(5,10)}=9.8, p<10^{-3}\right)$. In contrast to the control group, the $\mathrm{Rh}$ group was insensitive to reward size in the uncued task (Fig. $4 C, D$; reward size, $F_{(3,6)}=0.67, p=0.6$, accumulated reward, $\left.F_{(5,10)}=0.4, p=0.84\right)$. Direct comparison between the control and $\mathrm{Rh}$ groups revealed a significant effect of Rh lesions on performance (group, $F_{(1,4)}=3.1, p=0.15$; reward size $\times$ group, $\left.F_{(3,12)}=5.6, p=0.01\right)$. Furthermore, there was a significant difference in $\mathrm{Rh}$ group performance across the cued and uncued tasks (task, $F_{(1,2)}=21.9, p=0.04$; task $\times$ reward, $\left.F_{(3,6)}=5.3, p=0.04\right)$. Based upon this partial savings in the visually cued task, we conclude that monkeys were still able to discriminate among the various reward magnitudes, suggesting that the more severe impairment seen in the uncued task was not due to a low level sensory deficit.

The absence of sensory cues to reward in the uncued task suggests that control monkeys were using the block-wise task structure to predict forthcoming reward size (i.e., the most likely reward size on a given trial is the reward size received on the previous trial) and comparisons between predicted and previously experienced (i.e., at least one block prior) reward sizes to value predicted rewards. We wondered whether Rh lesions selectively disrupted a specific phase of this process of prediction and valuation, e.g., impairing performance early but sparing performance late in a block. To explore the dynamics of the monkeys' behavior we standardized release intervals and binned according to trial number within each block (see Materials and Methods, Data analysis).

Control monkeys rapidly adjusted their release interval following changes in reward size. Control responses tended to be slower or faster than average, according to whether the reward was small (1 drop) or large ( 8 drops), respectively, on the first trial after receiving information that reward size had changed ("shift trial," i.e., the second trial in a block; Fig. 5 control). There was a significant effect of withinblock trial number on control group performance (reward size $\times$ within-block trial, $F_{(72,144)}=1.76, p<10^{-3}$ ). In contrast to the control group, the $\mathrm{Rh}$ group was insensitive to within-block trial number, responding similarly regardless of reward size or within-block trial (Fig. $5 \mathrm{Rh}$; reward size $\times$ within-block trial, $\mathrm{F}_{72,144)}=$ $0.9, p=0.7)$. Comparison of the control and Rh groups revealed a significant effect of $\mathrm{Rh}$

Figure 5. Rh lesions impair performance across all phases of a block in the uncued task. Standardized release intervals (ordinate) for each reward size (separate color traces) are plotted versus within-block trial number (abscissa) for the control (left) and Rh (right) groups. The control but not Rh group rapidly adjusted their performance following block transitions before maintaining responding at a stable rate. There was a significant three-way interaction between lesion, reward size, and within-block trial. In all panels, error bars indicate SEM. RI, Release interval.

\section{Uncued reward size task}

In the uncued task, rewards were delivered for touching and releasing a bar, reward size varied randomly in a block-wise fashion ( 25 trials per block) and no visual cues predicted reward size nor instructed response. There was no difference between the control and $\mathrm{Rh}$ groups in the total number of lever presses in a session lesions on trial-by-trial performance (group, $F_{(1,4)}=0.7, p=0.44$; reward size $\times$ within-block trial $\times$ group, $\left.F_{(72,288)}=1.4, p=0.03\right)$. Thus, Rh lesions did not selectively impair performance within a specific phase of a block in the uncued task.

Finally, it is possible the depth of the impairment in the Rh group in this task is dependent upon the magnitude of the change 

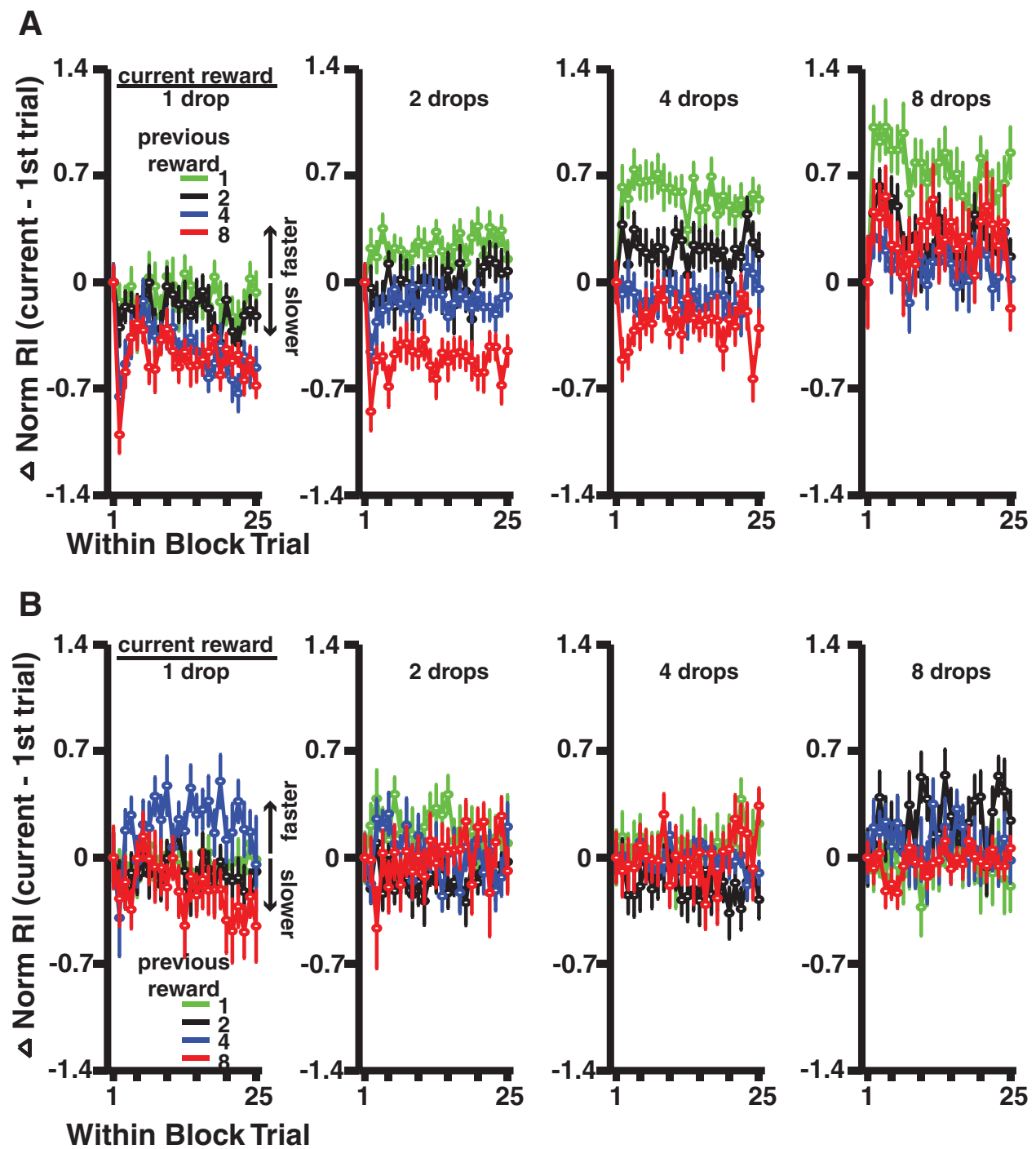

Figure 6. Effect of local reward contrast in the uncued task for all trials. $A$, In each panel, the average change in standardized release interval, normalized to the standardized response on the first trial (ordinate), is plotted against the within-block trial number (abscissa). Data were first sorted according to the reward size in the current block (1, 2, 4, or $8 \mathrm{drops}$, indicated above each panel) and then sorted according to the reward size in the previous block (separate colors, see key in $\boldsymbol{A}$ ). Data in $\boldsymbol{A}$ are from the control group. $\boldsymbol{B}$, Conventions as in $\boldsymbol{A}$ but for data from the Rh group. Norm, Normalized; Rl, release interval.

in reward size across blocks. For example, monkeys with Rh lesions might have been able to distinguish large ( 1 drop to 8 drops or 8 drops to 1 drop) but not small ( 2 drops to 4 drops or 4 drops to 2 drops) changes in reward size. To examine this possibility, we again analyzed the dynamics of the monkeys' performance, first binning standardized responses according to: (1) the reward size in the current block, (2) the reward size in the preceding block and, (3) within-block trial number, then normalizing to the response on the first trial in a block (Fig. 6A,B). To quantify the effect of the magnitude of the change in reward size across blocks on performance we correlated the difference in performance from the first to second trial in a block with the difference in reward size from the previous to the current block (Fig. $7 A, B$ ). There was a significant correlation between these measures for the control $\left(r=0.44, p<10^{-10}\right)$ but not the Rh $(r=0.04, p=$ $0.16)$ group.

In summary, varying reward size across blocks of uncued trials in a lever pressing task caused unoperated controls, but not monkeys with bilateral Rh removals, to modulate their rate of lever pressing, with faster (slower) responses occurring in large (small) reward blocks. As no visual cues predicted reward size in the uncued task, it follows that the Rh group's deficit in this task cannot be the result of difficulty in using visual cues to value predicted rewards.

\section{Discussion}

We found that bilateral removal of $\mathrm{Rh}$ altered the manner in which monkeys responded to two different cues to predicted reward value: (1) visual cues that signal specific reward sizes, and (2) changes in reward size across blocks of identical trials. These effects suggest that $\mathrm{Rh}$ is a critical part of the circuitry responsible for learning and updating the value of reinforcement; a function that is crucial for normal learning and motivation. Here, we situate these results with respect to previous work and discuss the implications of these findings for current theories of the organization of the neural substrates of memory and reward guided behavior.

Consistent with prior reports, we found that damage to Rh significantly impaired the ability to use visual cue-reward associations to guide behavior (Murray et al., 1998; Liu et al., 2000, 2004; Winters 
A
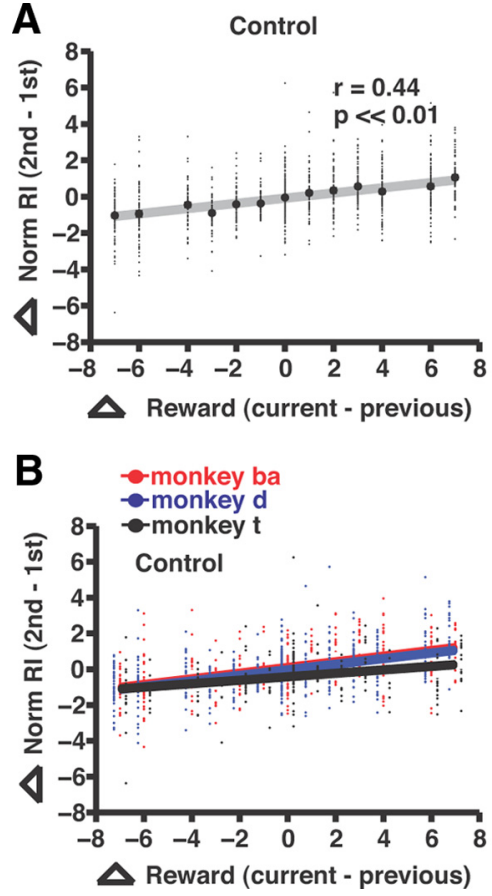

Rh
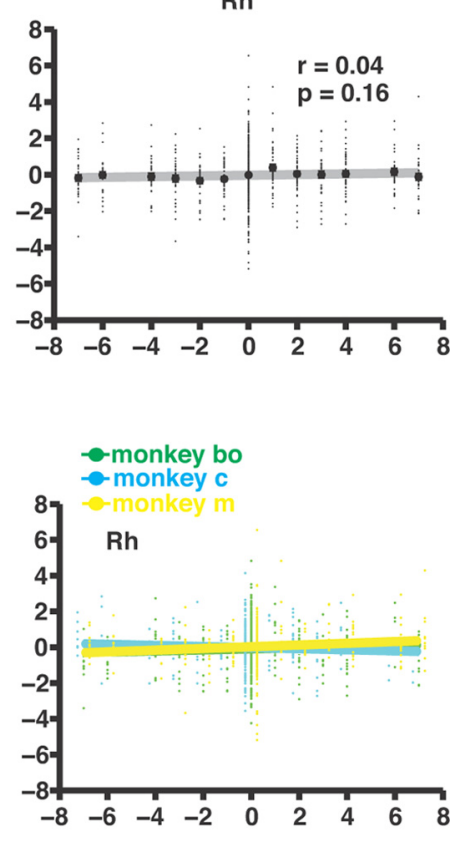

Figure 7. A, Effect of local reward contrast in the uncued task-shift trials. The change in normalized release interval (secondfirst trial in a block, ordinate) is plotted against the change in reward (current-previous block, abscissa) separately for the control (left) and Rh (right) groups. Solid lines are best fit linear regressions. The second trial in a block is the first trial in which the monkeys are aware of the change in reward size ("shift trial"). There was a significant correlation between the change in performance from first to shift trials and the magnitude of the change in reward size from the previous to the current block for the control but not the Rh group. $\boldsymbol{B}$, Conventions as in $\boldsymbol{A}$ but data are plotted separately for each monkey in the control (left) and Rh (right) groups. Norm, Normalized; Rl, release interval.

et al., 2010). Unlike in the work of Liu et al. (2000; 2004), in which visual cues signaled the number of trials remaining to reward in schedules of multiple red-green color discrimination trials, our group of monkeys retained some sensitivity to reward value in our visually cued task.

The Rh group's partial sensitivity to reward size in our visually cued task indicates both that the Rh group was able to discriminate reward magnitudes and able to use predictive cues to estimate reward value, albeit differently than controls. This latter function could have been supported by processing in other structures that signal cue-reward associations, such as the amygdala (Sugase-Miyamoto and Richmond, 2005), the ventral striatum (Bowman et al., 1996) and the orbitofrontal cortex (Simmons and Richmond, 2008). However, because these distant structures were intact in both the present case and in the work of Liu et al. (2000), in which monkeys showed no sensitivity to reward value following Rh ablations, we suggest the savings observed in the visually cued reward size task is most likely due to a specific feature of this task.

Recently, it has been shown that intact monkeys are sensitive to temporal context in multitrial reinforcement schedules (La Camera and Richmond, 2008). Specifically, their performance of unrewarded trials improves with the number of previously completed unrewarded trials in a schedule. This suggests that monkeys combine information from previous trials and current trials when estimating the value of completing unrewarded trials, an effect known within the economic literature as "framing" (Tversky and Kahneman, 1981). This framing effect is absent from our visually cued task, in which every trial is rewarded and predicted value is determined solely by the amount of primary reinforcement signaled by the visual cue. Thus, the more severe impair- ment in the multitrial reinforcement schedule task following Rh ablationsrelative to what we observed in our visually cued reward size task-could be the result of the greater mnemonic demands of integrating information over multiple trials. Our uncued task, in which predicted value estimates are crucially dependent upon information from previous trials, represents a strong test of this hypothesis.

Control group performance in the uncued task suggests that monkeys were using both local (current versus previous trial) and global (current versus all potential reward sizes) reward context to value predicted rewards. Evidence for a local comparison comes from trials in which information about changes in reward size is first available ("shift trials"). The magnitude of the change in the standardized release interval on shift trials was significantly correlated with the magnitude of the change in reward size (current-previous block; refer to Fig. $7 A$ ). If control monkeys were using the reward size earned on the previous trial to instruct their upcoming response, we would have expected these sharp changes in release intervals for trials immediately following block transitions. However, if the previous trial's reward size is the only information that monkeys were using to adjust their behavior, we would have expected no differences in release interval across reward sizes for the remaining trials in a block. This was not the case (refer to Fig. 5). After a period of 5-10 trials release intervals were consistently ordered according to reward size (that is, responses went from slow to fast as drop size increases from small to large, but did not change much trial-by-trial). This consistent ordering of release intervals according to reward size suggests a stable longterm valuation of the various reward sizes, one that is likely to be based upon the range of available options (Kobayashi et al., 2010; Louie et al., 2011).

A number of studies have reported encoding of cue-reward associations, discrepancies between expected and received rewards, and the subjective value of rewards in a network of brain regions including: midbrain dopamine neurons (Schultz et al., 1993; Tobler et al., 2005), the amygdala (Gottfried et al., 2003; Paton et al., 2006), medial and orbital prefrontal cortex (Tremblay and Schultz, 1999; Cox et al., 2005; Bouret and Richmond, 2010) and the striatum (Hollerman et al., 1998; Elliot et al., 2000; Lau and Glimcher, 2008). Rh is reciprocally connected with many of these areas (Akil and Lewis, 1993; Goldsmith and Joyce, 1996; Saleem et al., 2008). When viewed in light of these connections, our results suggest that Rh ought to be seen as a key component of this reward circuit. Furthermore, both the severe impairment in our uncued task produced by Rh lesions, and previous work demonstrating that $\mathrm{Rh}$ is a key part of the medial temporal lobe (MTL) memory system (for a review see Squire et al., 2004) suggest that the function of Rh in this system is integration of the history of previous rewards, i.e., the particular reward context that a predicted reward is delivered within, to estimate predicted reward value. 
Context often refers to a particular environment (Kim and Fanselow, 1992), the particular identity and arrangement of stimuli in an environment (González et al., 2003) or a particular arrangement of movements in a sequential motor act (Clegg et al., 1998). All of these different types of context can serve as cues to behavior, both to generate expectations about outcomes and plan responses. In the present case, both the visually cued and uncued tasks involved several stimuli and actions. These bore a constant relationship to one another and were reliably associated with reward, regardless of reward size (e.g., lever pressing, background images on the display monitor, the behavioral testing chamber, etc.). Thus, neither task lacked individual cue-reward nor context-reward associations. However, only in the visually cued task was there a specific visual cue predicting the value of the forthcoming reward. Similarly, only in the uncued task were monkeys required to estimate predicted reward value based upon a comparison between expected and previously earned rewards. This lack of a specific visual cue to reward value and a requirement for integrating information over a longer timescale to predict reward value suggests the uncued task placed a greater mnemonic demand on the monkeys.

Evidence for a role of the MTL in normal learning and memory has been accumulating since early studies of the patient H.M. (Scoville and Milner, 1957). However, the role of distinct MTL subregions in encoding various aspects of a memory remains an open question. There is agreement about the importance of the hippocampus and parahippocampal cortex for spatial memory and spatial context (Squire et al., 2004). More recently, work demonstrating that $\mathrm{Rh}$ lesions yield deficits, in rodents and monkeys, in tasks that require discrimination of context have led to the hypothesis that $\mathrm{Rh}$ is primarily important for flexibly manipulating the contextual relationships between sensory items held in memory (Bucci et al., 2000; Buckmaster et al., 2004). The profound impairment observed here suggests that $\mathrm{Rh}$ is also critical for using the context in which a predicted reward occurs to estimate its value. The specific reward memory that this requires could be the sensory qualities of the primary reward, or some more abstract value representation. Rh receives input from numerous areas implicated in encoding both the perceptual dimensions of primary rewards and higher level value representations (Saleem et al., 2008).

As the primary relay between the hippocampus and neocortex, $\mathrm{Rh}$ is uniquely situated to combine different types of information surrounding a specific event for consolidation into long term memory (de Curtis and Paré, 2004). Associating temporally discontinuous events is a defining feature of episodic memory (Clayton et al., 2003). Thus, it is possible that connections between Rh and the temporal lobe (Saleem and Tanaka, 1996), and $\mathrm{Rh}$ and ventral and medial prefrontal cortex (Saleem et al., 2008), respectively, might be important for aggregating information about visual, reward and motor context in our tasks into distinct episodes. This proposal is supported by recent work highlighting the interdependence of episodic memory, and retrospection and prospection in general (Eichenbaum and Fortin, 2009), and enhanced connectivity between the prefrontal cortex and medial temporal lobe when estimating expected value requires imagining future episodes (Peters and Büchel, 2010). Further studies using disconnection techniques to selectively interrupt communication between $\mathrm{Rh}$ and its inputs from the prefrontal and temporal cortex will be needed to tease apart the contributions of particular brain regions and the pathways connecting them.

\section{References}

Akil M, Lewis DA (1993) The dopaminergic innervation of monkey entorhinal cortex. Cereb Cortex 3:533-550.

Bachevalier J, Beauregard M, Alvarado MC (1999) Long-term effects of neonatal damage to the hippocampal formation and amygdaloid complex on object discrimination and object recognition in rhesus monkeys (Macaca mulatta). Behav Neurosci 113:1127-1151.

Bouret S, Richmond BJ (2010) Ventromedial and orbital prefrontal neurons differentially encode internally and externally driven motivational values in monkeys. J Neurosci 30:8591-8601.

Bowman EM, Aigner TG, Richmond BJ (1996) Neural signals in the monkey ventral striatum related to motivation for juice and cocaine rewards. J Neurophysiol 75:1061-1073.

Brown MW, Aggleton JP (2001) Recognition memory: what are the roles of the perirhinal cortex and hippocampus? Nat Rev Neurosci 2:51-61.

Bucci DJ, Phillips RG, Burwell RD (2000) Contributions of postrhinal and perirhinal cortex to contextual information processing. Behav Neurosci 114:882-894.

Buckmaster CA, Eichenbaum H, Amaral DG, Suzuki WA, Rapp PR (2004) Entorhinal cortex lesions disrupt the relational organization of memory in monkeys. J Neurosci 24:9811-9825.

Burwell RD, Bucci DJ, Sanborn MR, Jutras MJ (2004) Perirhinal and postrhinal contributions to remote memory for context. J Neurosci 24:11023-11028.

Clayton NS, Bussey TJ, Dickinson A (2003) Can animals recall the past and plan for the future? Nat Rev Neurosci 4:685-691.

Clegg BA, Digirolamo GJ, Keele SW (1998) Sequence learning. Trends Cogn Sci 8:275-281.

Cox SM, Andrade A, Johnsrude IS (2005) Learning to like: a role for human orbitofrontal cortex in conditioned reward. J Neurosci 25:2733-2740.

de Curtis M, Paré D (2004) The rhinal cortices: a wall of inhibition between the neocortex and the hippocampus. Prog Neurobiol 74:101-110.

Eichenbaum H, Fortin NJ (2009) The neurobiology of memory based predictions. Philos Trans R Soc Lond B Biol Sci 364:1183-1191.

Eichenbaum H, Yonelinas AP, Ranganath C (2007) The medial temporal lobe and recognition memory. Annu Rev Neurosci 30:123-152.

Elliott R, Friston KJ, Dolan RJ (2000) Dissociable neural responses in human reward systems. J Neurosci 20:6159-6165.

Fritz J, Mishkin M, Saunders RC (2005) In search of an auditory engram. Proc Natl Acad Sci U S A 102:9359-9364.

Goldsmith SK, Joyce JN (1996) Dopamine D2 receptors are organized in bands in normal human temporal cortex. Neuroscience 74:435-451.

González F, Quinn JJ, Fanselow MS (2003) Differential effects of adding and removing components of a context on the generalization of conditional freezing. J Exp Psychol Anim Behav Process 29:78-83.

Gottfried JA, O'Doherty J, Dolan RJ (2003) Encoding predictive reward value in human amygdala and orbitofrontal cortex. Science 301:1104-1107.

Goulet S, Murray EA (2001) Neural substrates of crossmodal association memory in monkeys: the amygdala versus the anterior rhinal cortex. Behav Neurosci 115:271-284.

Hollerman JR, Tremblay L, Schultz W (1998) Influence of reward expectation on behavior-related neuronal activity in primate striatum. J Neurophysiol 80:947-963.

Kim JJ, Fanselow MS (1992) Modality specific retrograde amnesia of fear. Science 256:675-677.

Kobayashi S, Pinto de Carvalho O, Schultz W (2010) Adaptation of reward sensitivity in orbitofrontal neurons. J Neurosci 30:534-544.

La Camera G, Richmond BJ (2008) Modeling the violation of reward maximization and invariance in reinforcement schedules. PLoS Comput Biol 4:e1000131.

Lau B, Glimcher PW (2008) Value representations in the primate striatum during matching behavior. Neuron 58:451-463.

Liu Z, Murray EA, Richmond BJ (2000) Learning motivational significance of visual cues for reward schedules requires rhinal cortex. Nat Neurosci 3:1307-1315.

Liu Z, Richmond BJ, Murray EA, Saunders RC, Steenrod S, Stubblefield BK, Montague DM, Ginns EI (2004) DNA targeting of rhinal cortex D2 receptor protein reversibly blocks learning of cues that predict reward. Proc Natl Acad Sci U S A 101:12336-12341.

Louie K, Grattan LE, Glimcher PW (2011) Reward value-based gain control: divisive normalization in parietal cortex. J Neurosci 31:10627-10639. 
Lowe CF, Davey GC, Harzem P (1974) Effects of reinforcement magnitude on interval and ratio schedules. J Exp Anal Behav 22:553-560.

Meunier M, Bachevalier J, Mishkin M, Murray EA (1993) Effects on visual recognition of combined and separate ablations of the entorhinal and perirhinal cortex in rhesus monkeys. J Neurosci 13:5418-5432.

Minamimoto T, La Camera G, Richmond BJ (2009) Measuring and modeling the interaction among reward size, delay to reward, and satiation level on motivation in monkeys. J Neurophysiol 101:437-447.

Moser EI, Kropff E, Moser MB (2008) Place cells, grid cells and the brain's spatial representation system. Annu Rev Neurosci 31:69-89.

Mumby DG, Pinel JP (1994) Rhinal cortex lesions and object recognition in rats. Behav Neurosci 108:11-18.

Murray EA, Gaffan D, Mishkin M (1993) Neural substrates of visual stimulusstimulus association in rhesus monkeys. J Neurosci 13:4549-4561.

Murray EA, Baxter MG, Gaffan D (1998) Monkeys with rhinal cortex damage or neurotoxic hippocampal lesions are impaired on spatial scene learning and object reversals. Behav Neurosci 112:1291-1303.

Murray EA, Bussey TJ, Saksida LM (2007) Visual perception and memory: a new view of medial temporal lobe function in primates and rodents. Annu Rev Neurosci 30:99-122.

Parker A, Gaffan D (1998) Lesions of the primate rhinal cortex cause deficits in flavour-visual associative memory. Behav Brain Res 93:99-105.

Paton JJ, Belova MA, Morrison SE, Salzman CD (2006) The primate amygdala represents the positive and negative value of visual stimuli during learning. Nature 439:865-870.

Perone M, Courtney K (1992) Fixed-ratio pausing: Joint effects of past reinforcer magnitude and stimuli correlated with upcoming magnitude. J Exp Anal Behav 57:33-46.

Peters J, Büchel C (2010) Episodic future thinking reduces reward delay discounting through an enhancement of prefrontal-mediotemporal interactions. Neuron 66:138-148.

Saleem KS, Tanaka K (1996) Divergent projections from the anterior inferotemporal area TE to the perirhinal and entorhinal cortices in the macaque monkey. J Neurosci 16:4757-4775.

Saleem KS, Kondo H, Price JL (2008) Complementary circuits connecting the orbital and medial prefrontal networks with the temporal, insular, and opercular cortex in the macaque monkey. J Comp Neurol 506:659-693.

Sauvage MM, Beer Z, Ekovich M, Ho L, Eichenbaum H (2010) The caudal medial entorhinal cortex: a selective role in recollection-based recognition memory. J Neurosci 30:15695-15699.

Schultz W (2007) Multiple dopamine functions at different time courses. Annu Rev Neurosci 30:259-288.

Schultz W, Apicella P, Ljungberg T, Romo R, Scarnati E (1993) Rewardrelated activity in the monkey striatum and substantia nigra. Prog Brain Res 99:227-235.

Scoville WB, Milner B (1957) Loss of recent memory after bilateral hippocampal lesions. J Neurol Neurosurg Psychiatry 20:11-21.

Simmons JM, Richmond BJ (2008) Dynamic changes in representations of upcoming and preceding reward in monkey orbitofrontal cortex. Cereb Cortex 18:93-103.

Squire LR, Stark CE, Clark RE (2004) The medial temporal lobe. Annu Rev Neurosci 27:279-306.

Sugase-Miyamoto Y, Richmond BJ (2005) Neuronal signals in the monkey basolateral amygdala during reward schedules. J Neurosci 25:11071-11083.

Tobler PN, Fiorillo CD, Schultz W (2005) Adaptive coding of reward value by dopamine neurons. Science 307:1642-1645.

Tremblay L, Schultz W (1999) Relative reward preference in primate orbitofrontal cortex. Nature 398:704-708.

Tversky A, Kahneman D (1981) The framing of decisions and the psychology of choice. Science 211:453-458.

Williams DC, Saunders KJ, Perone M (2011) Extended pausing by humans on multiple fixed-ratio schedules with varied reinforcer magnitude and response requirements. J Exp Anal Behav 95:203-220.

Winters BD, Bartko SJ, Saksida LM, Bussey TJ (2010) Muscimol, AP5, or scopolamine infused into perirhinal cortex impairs two-choice visual discrimination learning in rats. Neurobiol Learn Mem 93:221-228.

Yonelinas AP, Kroll NE, Dobbins I, Lazzara M, Knight RT (1998) Recollection and familiarity deficits in amnesia: convergence of remember-know, process dissociation, and receiver operating characteristic data. Neuropsychology 12:323-339. 